Supporting Information

\title{
A Humidity-controllable, High-Throughput, and Portable Nanofibrous Filter Coating System for Improving Air Quality
}

Yeon-Do Jeong, Chan Park, HyongUk Kim, Cheol Jeong Park, Byeongjun Lee, Jeongmin Kim and Seong J. Cho *

School of Mechanical Engineering, Chungnam National University, 99 Daehak-ro, Yuseong-gu,

Daejeon 34134, Korea; * Correspondence: scho@cnu.ac.kr; Tel.: +82-42-821-5648 
a

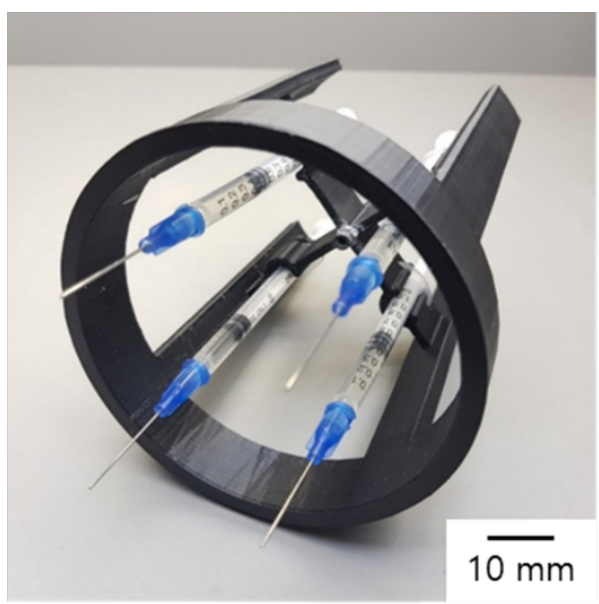

b

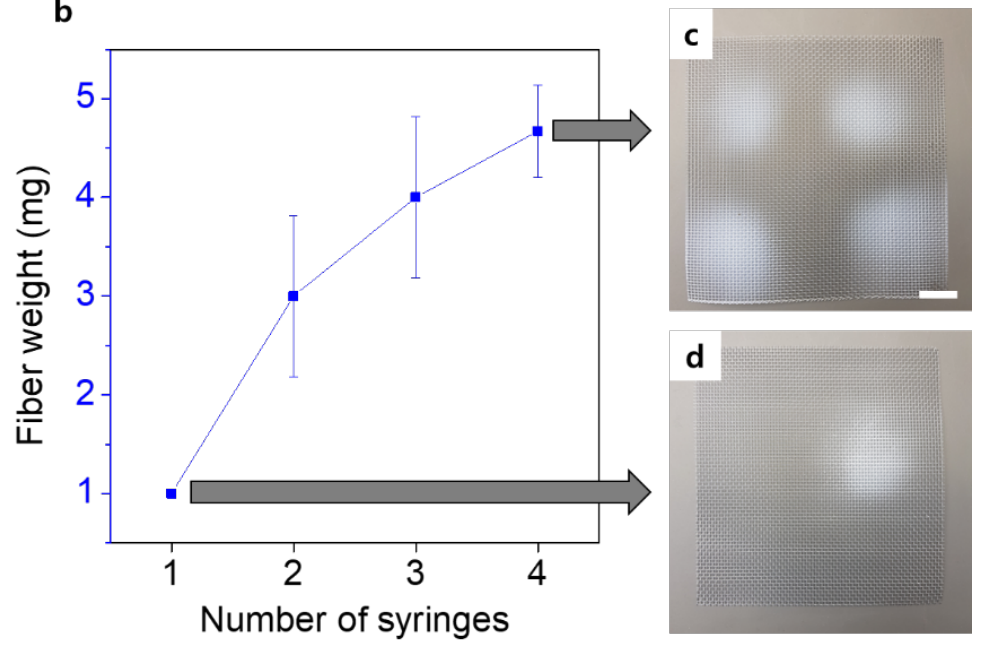

Figure S1 a) Holder mounted 4 syringes, b) Fiber weight versus number of syringes, c) Nanofibrous filters coated with 4 syringes (scale bar: $2 \mathrm{~cm}$ ) and d) with 1 syringe 

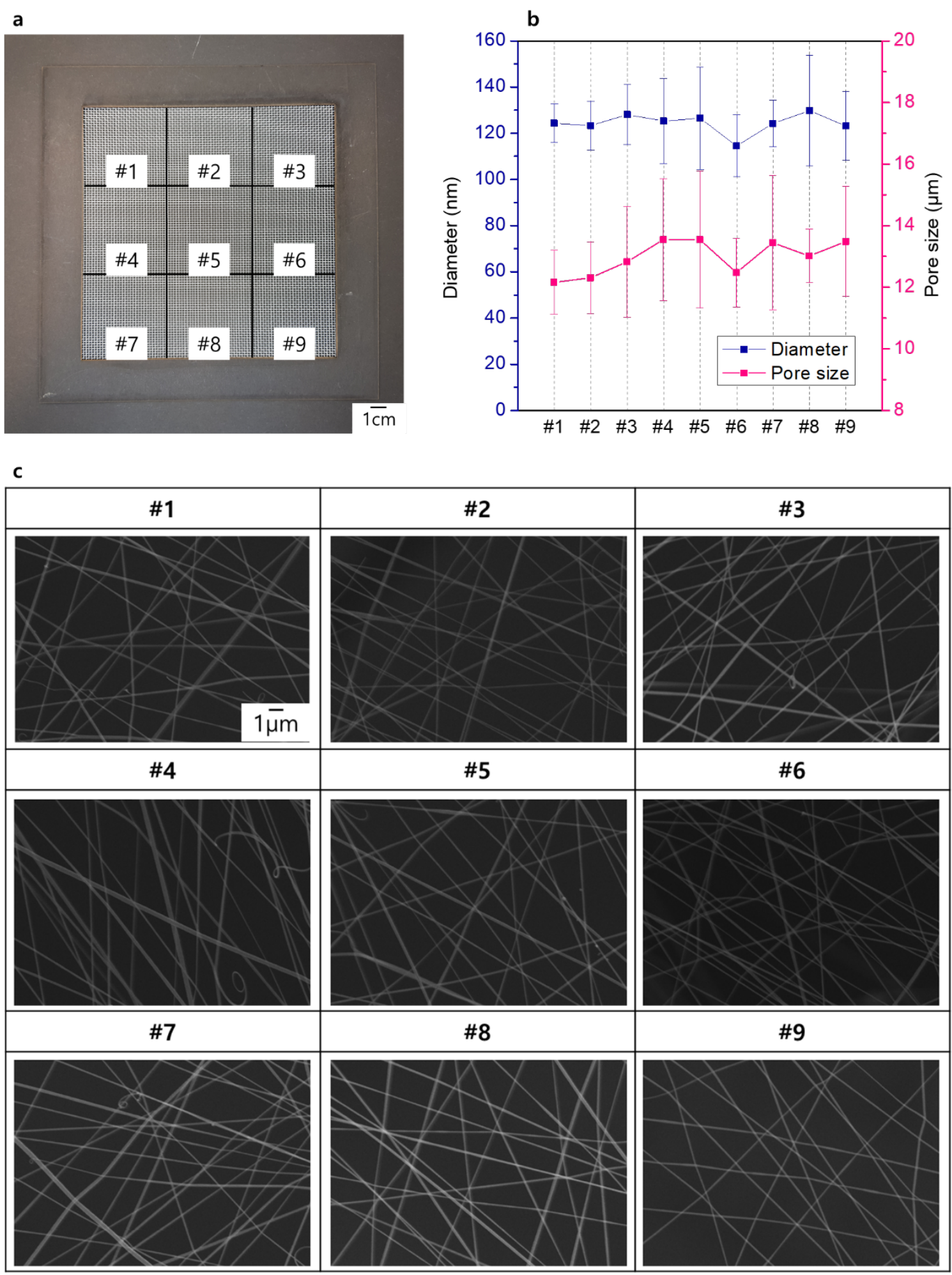

Figure S2 Filter Uniformity Test: a) Filter-coated window screen $(15 \mathrm{~cm} \mathrm{x} 15 \mathrm{~cm})$ divided into nine zones (\#1 \#9), b) Diameter and pore size of each zones, c) SEM images of each zones 

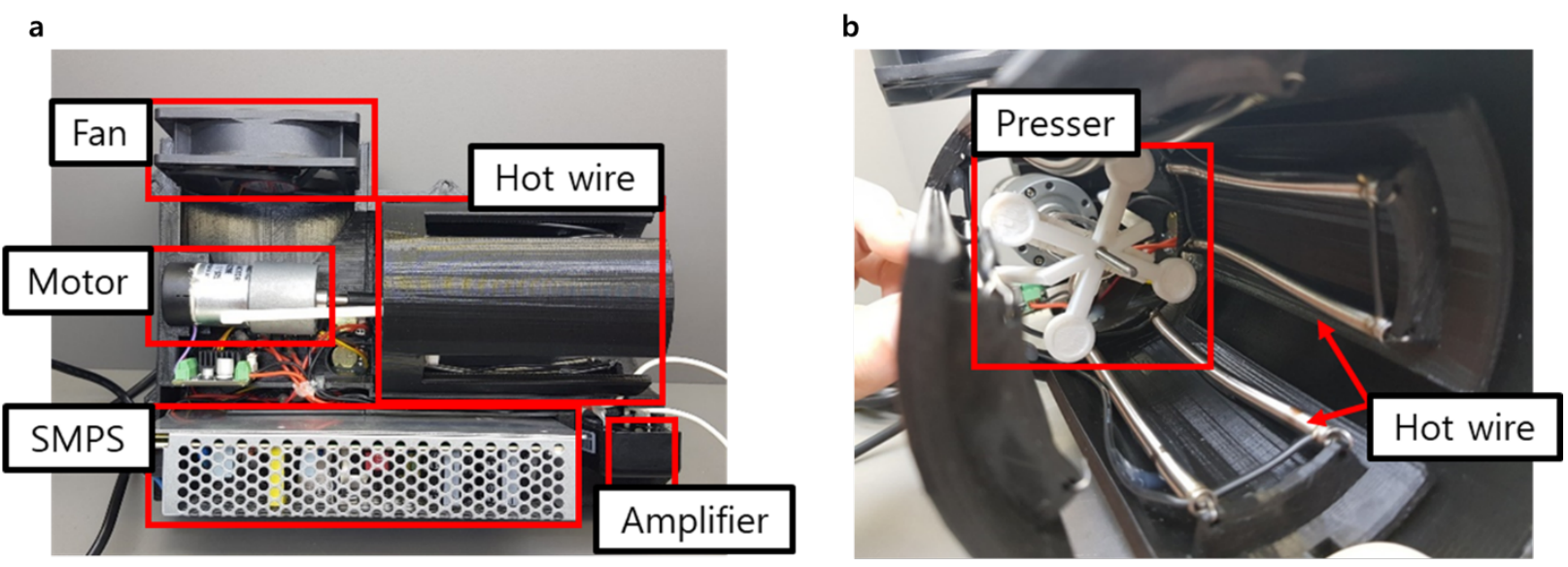

Figure S3 a) Inside view of the PEFCS, b) Inside view of the hot wire part 


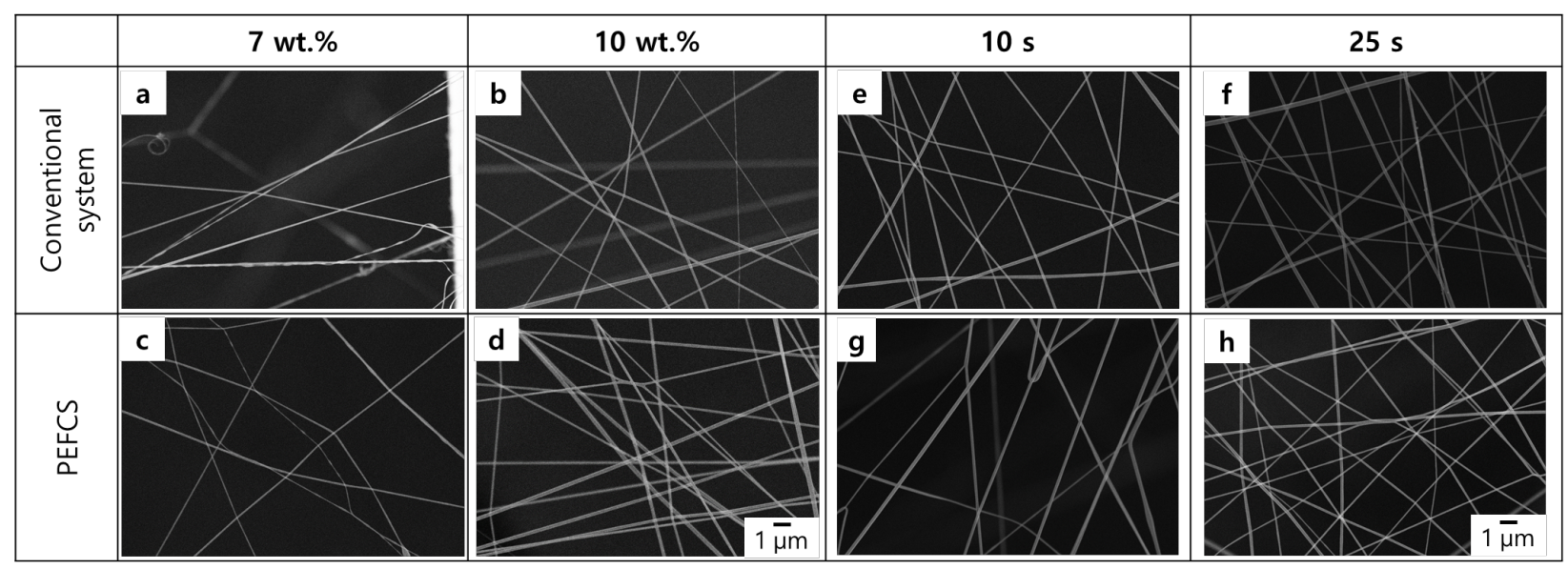

Figure S4 SEM images of electrospun nanofibrous filters fabricated by a conventional system and the PEFCS, a, c) 7 wt.\% and b, d) 10 wt.\% solution for e, g) $10 \mathrm{~s}$ and f, h) $25 \mathrm{~s}$ by a conventional system and the PEFCS, respectively 
a

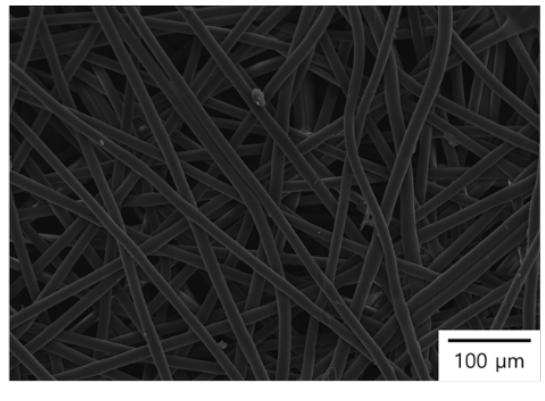

b

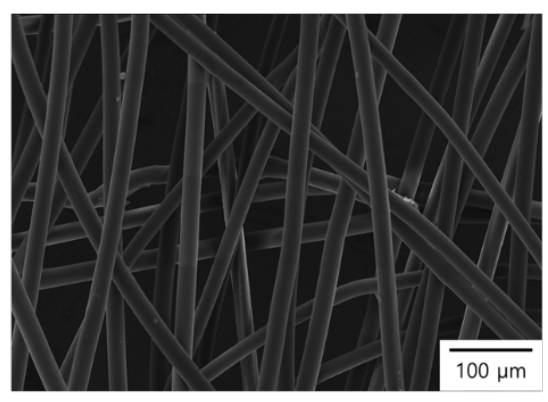

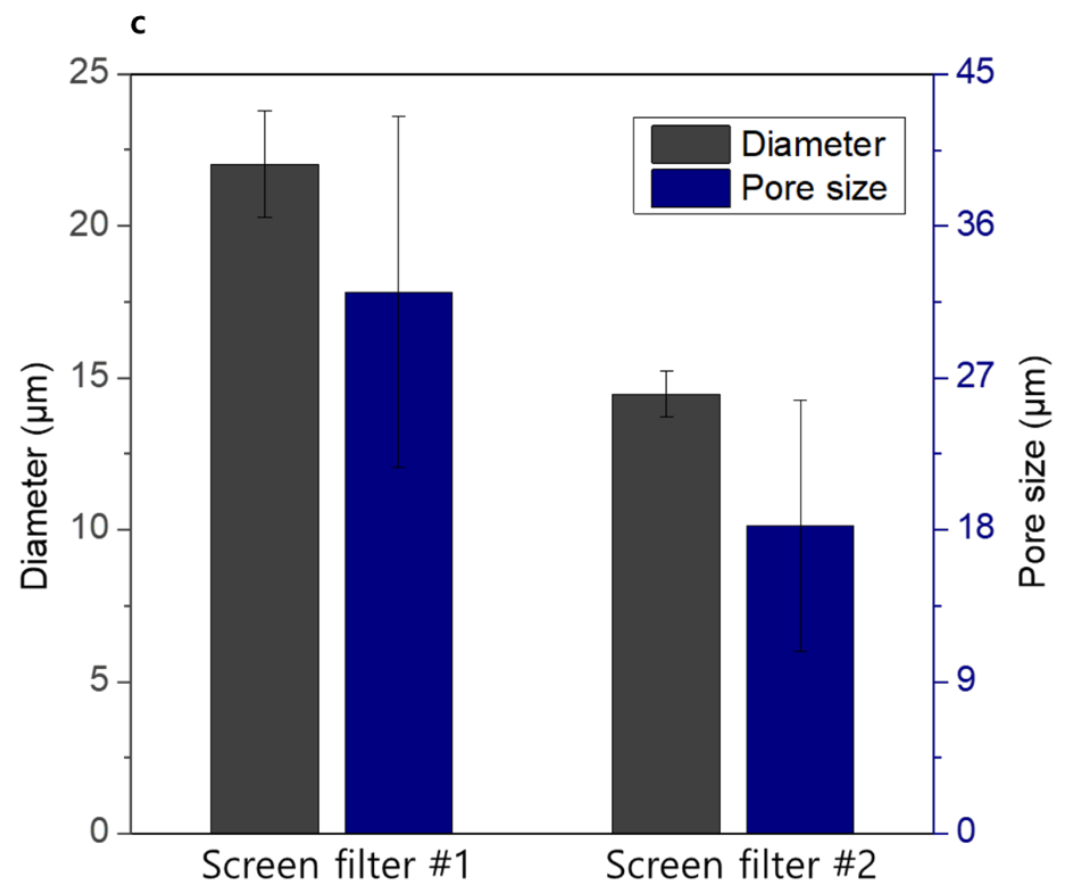

Figure S5 SEM images and morphological analysis of commercial screen filters a) SEM image of screen filter \#1, and b) screen filter \#2, c) morphological analysis of screen filters 